\title{
Dynamical models of love with time-varying fluctuations
}

\author{
J. Wauer ${ }^{\mathrm{a}, *}$, D. Schwarzer ${ }^{\mathrm{a}}$, G.Q. Cai ${ }^{\mathrm{b}}$, Y.K. Lin ${ }^{\mathrm{b}}$ \\ ${ }^{a}$ Institut für Technische Mechanik, Universität Karlsruhe (TH), Kaiserstraße 12, D-76128 Karlsruhe, Germany \\ ${ }^{\mathrm{b}}$ Center of Applied Stochastics Research, Florida Atlantic University, USA
}

\begin{abstract}
Human romantic relationships are studied via system dynamics methodology. Starting point is a time-invariant linear model of two individuals without interaction with environment. Specifically, time-dependent fluctuations both in the source terms and the system parameters are introduced and examined in their consequences where also more realistic nonlinear modeling is proposed and analyzed.
\end{abstract}

(c) 2006 Elsevier Inc. All rights reserved.

Keywords: Models of love; First-order differential equations; Nonlinear return functions; Time-varying source terms; Time-varying system parameters; Periodic and stochastic time fluctuations; Analytical results and simulation

\section{Introduction}

Strogatz [9] first suggested in a 1-page contribution and later in his book [10] analyzed love affairs via differential equations. ${ }^{1}$ This unusual approach was picked up by an Italian-Austrian group $[3,5,6]$ for a serious try to model such relationships by simple mathematical models composed of ordinary differential equations but under a little more realistic circumstances. Recently another attempt [7] was made to explain also the dynamics of love triangles. Related discrete dynamical models have recently been proposed in [2].

In the present contribution, we follow the differential equation approach once more. After reviewing the time-invariant linear model, a nonlinear model with more realistic bounded return functions is proposed. Special attention will be focused on the influence of time-varying source terms and time-varying system parameters. $^{2}$ To show the difference between earlier and new results clearly, we start with the case of constant source terms and constant system parameters (explanation and results) and then go to the model with time-varying source terms and system parameters, both periodic and stochastic ones.

Finally, an outlook for treating the more complicated case of love triangles is addressed.

\footnotetext{
* Corresponding author.

E-mail address: wauer@itm.uni-karlsruhe.de (J. Wauer).

1 Essentially the same model was described earlier by Rapoport [4] but concretely for the arms race of two countries.

${ }^{2}$ In a recent paper by Sprott [8] he addresses time-dependent source terms but he gives two examples only not examining them systematically.
} 


\section{Classical linear and nonlinear models of love}

We begin with the linear model of Strogatz [9] for two individuals (also a starting point in [7]), but with an additional constant source term representing the appeal (or repulsion if negative), as suggested in [5]:

$$
\begin{aligned}
& \frac{\mathrm{d} R}{\mathrm{~d} t}=a R+b J+f, \\
& \frac{\mathrm{d} J}{\mathrm{~d} t}=c R+d J+g .
\end{aligned}
$$

Specifically, $R(t)$ is the love (or hate) of individual 1 for individual 2, $J(t)$ is the love (or hate) of individual 2 for individual 1 . The coefficients $a$ and $b$ specify the "romantic style" of individual 1 , and $c$ and $d$ that of individual 2. For example, parameter $a$ describes the extent to which person 1 is encouraged by his/her own feelings, and $b$ is the extent to which he/she is encouraged by the feelings of person 2. $f$ expresses how person 2 appeals to person 1, and $g$ how person 1 appeals to person 2. The resulting dynamics is two-dimensional, governed by the corresponding initial conditions and the four system parameters $a, b, c$ and $d$ as well the two input terms $f$ and $g$. All the parameters are assumed to be constant in these two equations. It is entertaining, to classify the romantic styles for a specific pair of lovers depending on the signs of $a, b, c$ and $d$, and also to contemplate the romantic forecast for the various pairings, influenced by the sign and the magnitude of $f$ and $g$ as well the initial conditions. As noted by Sprott [7], the above modeling of Rinaldi [5] is more realistic since it allows feelings to grow from a state of indifference and provides an equilibrium not characterized by complete apathy.

As mentioned by Strogatz [9], the simplest model of the ill-fated romance between Romeo and Juliet is one with

$$
a=0, \quad b<0, \quad f=0, \quad c>0, \quad d=0, \quad g=0 .
$$

It describes the essence as follows: Juliet is in love with Romeo, but Romeo is a fickle lover in the presented version of the story. The more Juliet loves him, the more he begins to dislike her. But when she loses interest, his feelings for her warm up. She, on the other hand, tends to echo him. Her love grows when he loves her, and turns to hate when he hates her. The governing equations for their love are those of undamped oscillators, and the sad outcome of their affair is, of course, a never-ending cycle of love and hate. At the very least, they manage to achieve simultaneous love only during one-quarter of the time.

\subsection{Cautious lovers in linear formulation}

As introduced by the other papers mentioned above, the model used in the present discussion is a minimal one with the lowest possible number of state variables, namely, only the interaction between the two persons are taken into account, while the rest of the world does not participate in the love dynamics. In contrast to some of the other papers discussing all possible exotic combinations in the sign of system parameters, we deal with the most generic situation of such couples which are composed of cautious individuals (see [5,6]). The signs of all parameters will be fixed according to the phenomena oblivion, return and instinct according to [5]. The oblivion describes the forgetting process and gives rise to a loss of interest in the partner. It explains, for example, the typical exponential decay of the love $R$ of individual 1 for individual 2 which takes place after the death or estrangement of individual 2 (i.e., $a, d<0$ ). In contrast, the return and the instinct are sources of interest. More precisely, the return, i.e., the pleasure of being loved increases with the love of the partner (i.e., $b, c>0$ ) while the instinct is sensitive only to the partners appeal (physical, intellectual, financial, etc.) (i.e., $f, g>0$ ). Since both oblivion and return depend on one state variable only, synergism is assumed to be negligible. Very significant is the assumption that all factors of the relationship (typical for linear systems) are independent from each other (assumed to be true within the nonlinear modeling, too). In addition, the personalities and the appeals of the individuals do not vary in time, which rules out, for instance, the longertermed aging, learning and adaption processes, for instance, as well as daily or weekly activities affecting fast fluctuations of feelings. 
It follows that the starting point for the model of pair of cautious lovers is described by the equations

$$
\begin{aligned}
& \frac{\mathrm{d} x_{1}}{\mathrm{~d} t}=-\alpha_{10} x_{1}+\beta_{10} x_{2}+F_{10}, \\
& \frac{\mathrm{d} x_{2}}{\mathrm{~d} t}=-\alpha_{20} x_{2}+\beta_{20} x_{1}+F_{20},
\end{aligned}
$$

where all parameters $\alpha_{i 0}, \beta_{i 0}$ and $F_{i 0}, i=1,2$, are constant and positive (for simplification, we do not split the terms $F_{i 0}$ into appeal and reactiveness coefficient as in [5]). The terms of $\beta_{i 0} x_{j}$ are called the return functions, and $\beta_{i 0}$ are called the reaction parameters. The linear model (4) and (5) can be written for the state vector $\boldsymbol{x}=\left[x_{1}, x_{2}\right]^{\mathrm{T}}$ in the standard form $\dot{\boldsymbol{x}}=\boldsymbol{A} \boldsymbol{x}+\boldsymbol{u}$ where dots denote derivatives with respect to time and

$$
\boldsymbol{A}=\left[\begin{array}{cc}
-\alpha_{10} & \beta_{10} \\
\beta_{20} & -\alpha_{20}
\end{array}\right], \quad \boldsymbol{u}=\left[\begin{array}{c}
F_{10} \\
F_{20}
\end{array}\right] .
$$

Such a system is sometimes called positive because the matrix $\boldsymbol{A}$ has nonnegative off-diagonal elements and the vector $\boldsymbol{u}$ has positive components. It is assumed that they are completely indifferent to each other when they meet the first time, i.e., $\boldsymbol{x}(0)=\mathbf{0}$.

Under the assumptions of linearity, the system parameters have to fulfill the condition

$$
\beta_{10} \beta_{20}<\alpha_{10} \alpha_{20}
$$

to make the positive system asymptotically stable. If this were not the case, i.e. $\beta_{10} \beta_{20} \geqslant \alpha_{10} \alpha_{20}$, then the system would become unstable, corresponding to unrealistic unbounded feelings. However, for nonlinear model, (7) is not a necessary condition, which will be discussed later. The characteristic properties of the linear model are discussed (and proven) in detail in [5], and they are reviewed as follows for completeness.

Under condition (7), a cyclic behavior is not possible for a linear model with constant parameters. The equilibrium point $\overline{\boldsymbol{x}}^{\mathrm{T}}=\left[\bar{x}_{1}, \bar{x}_{2}\right]$ can be found to be

$$
\bar{x}_{1}=\frac{\alpha_{20} F_{10}+\beta_{10} F_{20}}{\alpha_{10} \alpha_{20}-\beta_{10} \beta_{20}}, \quad \bar{x}_{2}=\frac{\alpha_{10} F_{20}+\beta_{20} F_{10}}{\alpha_{10} \alpha_{20}-\beta_{10} \beta_{20}} .
$$

Eq. (8) shows that $\bar{x}_{i}>0, i=1,2$. Thus, beginning with zero initial conditions, $x_{1}(t) \geqslant 0$ and $x_{2}(t) \geqslant 0$ for all $t>0$, finally reach a steady-state $\overline{\boldsymbol{x}}$. This means that in the case of cautious lovers with positive appeals, they will never become antagonists, i.e., at least one of $x_{i}(t)$ is negative (hate).

Fig. 1 shows the dynamic behavior of the system with parameter values of $\alpha_{10}=2, \alpha_{20}=\beta_{10}=\beta_{20}=1$, $F_{10}=2, F_{20}=1$. If two individuals meet for the first time assuming $\boldsymbol{x}(0)=\mathbf{0}$, they will develop positive feelings tending toward the positive equilibrium value $\overline{\boldsymbol{x}}$. The system has positive eigenvalues only, i.e., the equilibrium of system (6) cannot be a focus. In other words, the transients of $x_{i}(t)$ cannot be damped oscillations, and they are strictly increasing under the zero initial conditions, i.e., $\dot{x}_{i}(t)>0, i=1,2$, for all $t>0$, as shown in Fig. 1 . However, for nonzero initial conditions, one of two variables $x_{i}(t)$ can first decrease and then increase (see trajectory $A \rightarrow \overline{\boldsymbol{x}}$ in Fig. 1) or vice versa (see trajectory $B \rightarrow \overline{\boldsymbol{x}}$ ). Assume that a couple is at equilibrium and that individual 2 has, for some reason, a sudden decline of interest in the partner. The consequence is that individual 1 will suffer during the whole transient bringing the couple back to equilibrium, namely, $x_{1}$ decreases along the trajectory $A \rightarrow \overline{\boldsymbol{x}}$.

It is known that the system generally has two different exponential decay rates, characterized by two dominant time constants, when approaches to the equilibrium. The two straight trajectories are extreme cases where the two functions $\left(x_{i}(t)-\bar{x}_{i}\right), i=1,2$ decay exponentially at the same rate. The slowest decay occurs along a trajectory which has a positive slope, the fastest decay along the other trajectory with negative slope. Two further remarkable properties are also revealed. First, an increase in the reaction parameter $\beta_{i 0}$ and the appeal term $F_{i 0}$ of individual $i$ gives rise to an increase in the love of both individuals at equilibrium, i.e., larger $\bar{x}_{i}$ values. Secondly, an increase in $\beta_{10}$ or $\beta_{20}$ causes an increase of one dominant time constant and an decrease of another. When $\beta_{10} \beta_{20}$ approaches $\alpha_{10} \alpha_{20}$, one tends to infinity and another tends to $1 /\left(\alpha_{10} \alpha_{20}\right)$.

Summarizing all these properties, the romantic relationship between cautious lovers can easily be described. First, individuals with positive appeal are capable of establishing a steady love relationship. The emotional pattern of two cautious people falling in love is quite regular: beginning with complete indifference, then love growing continuously until a plateau is reached. The level of plateau is higher for couples with higher reaction 


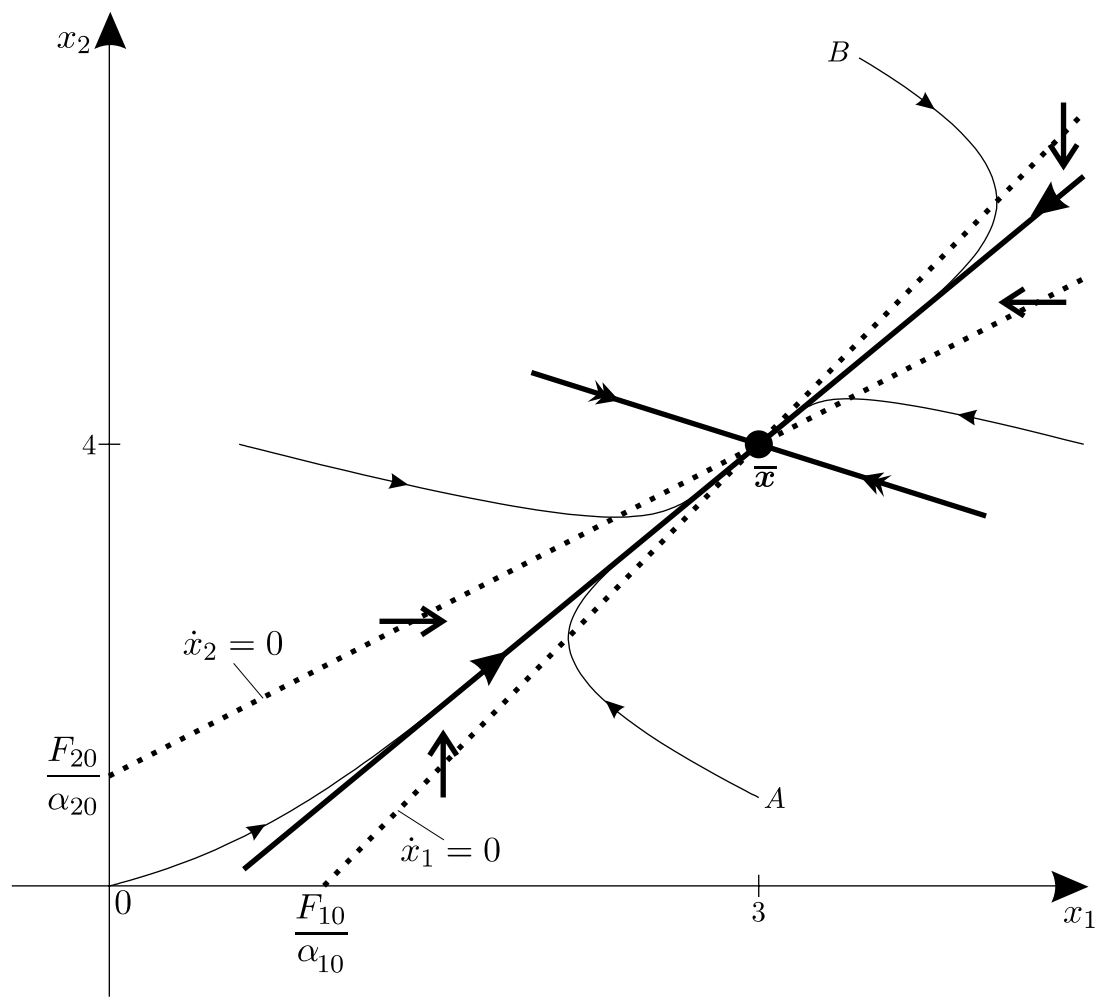

Fig. 1. Trajectories (continuous lines) and nullclines (dotted lines) of the system according to [5]. The straight trajectories are identified by the two eigenvectors.

and appeal, respectively. Finally, couples with high reaction respond promptly during the first phase of their romantic relationship, but are slow in reaching their plateau. Together with Eq. (8), this means that there is an inverse correlation between the time needed to reach the equilibrium and the final quality $\left(\bar{x}_{1}\right.$ and $\left.\bar{x}_{2}\right)$ of the relationship. Thus, passions that develop too quickly are associated with poor romantic relationships.

\subsection{Cautious lovers with nonlinear return functions}

In a next step, the linear return function $R_{i}=\beta_{i 0} x_{j}$ will be replaced more realistically for cautious lovers. Since an unbounded behavior cannot be realistic, it is more appropriate by assuming that, for positive values of $x_{j}$, the return function $R_{i}$ is positive, increasing, concave and bounded, and it is negative, increasing, convex and bounded for negative values of $x_{j}$. To meet these requirements, the point-symmetric models

$$
R_{1}=\beta_{10} \frac{x_{2}}{1+\varepsilon_{0}\left|x_{2}\right|}, \quad R_{2}=\beta_{20} \frac{x_{1}}{1+\varepsilon_{0}\left|x_{1}\right|}
$$

are proposed. Fig. 2 shows the corresponding graph. It is noted that monotonically increasing return functions are typical for cautious individuals.

In passing, we note that the boundedness of the return function is a property that must hold also for noncautious people, and it represents the psycho-physical mechanisms that prevent people from reaching dangerously high stresses. However, it may not always be monotonically increasing for noncautious individuals when pressure and involvement are too high.

The following mathematical model with constants $F_{i 0}, i=1,2$, will first be investigated, namely

$$
\begin{aligned}
& \dot{x}_{1}=-\alpha_{10} x_{1}+\beta_{10} \frac{x_{2}}{1+\varepsilon_{0}\left|x_{2}\right|}+F_{10}, \\
& \dot{x}_{2}=-\alpha_{20} x_{2}+\beta_{20} \frac{x_{1}}{1+\varepsilon_{0}\left|x_{1}\right|}+F_{20} .
\end{aligned}
$$




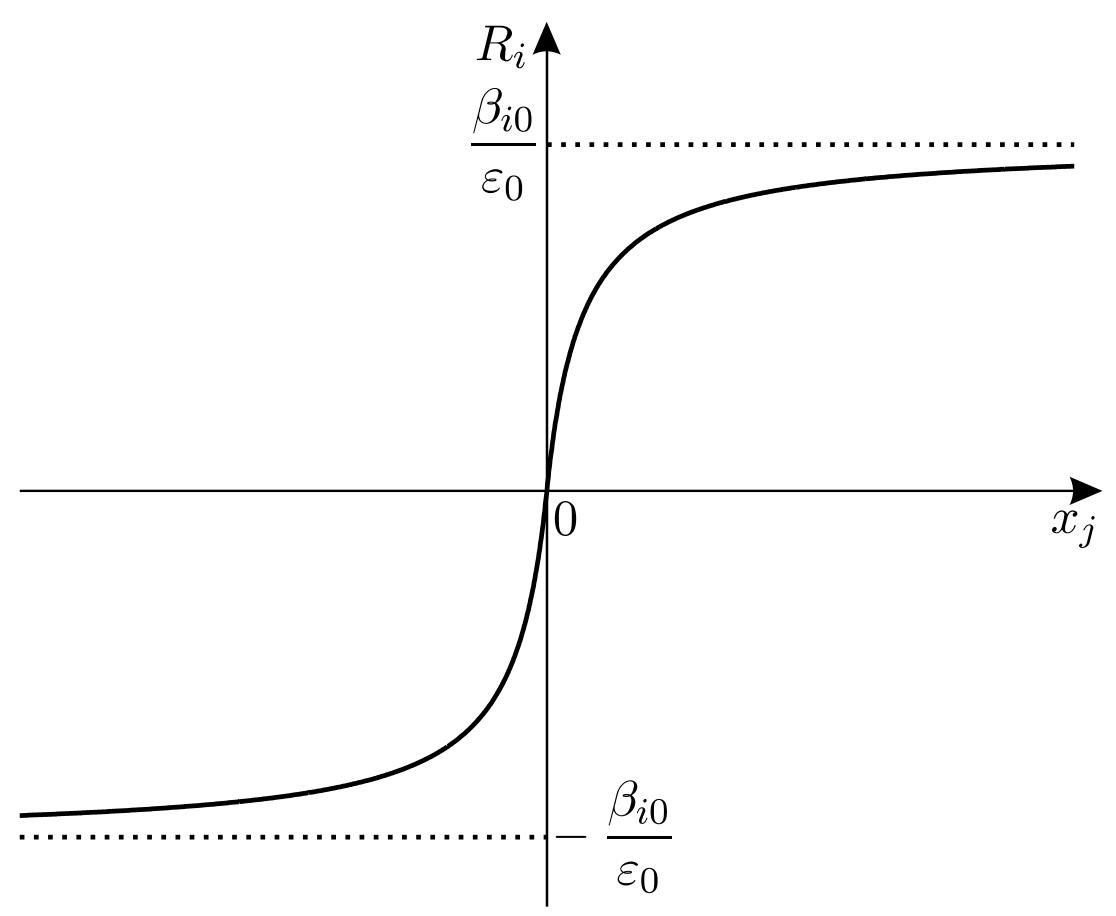

Fig. 2. Return function $R_{i}$ of cautious individuals.

To find the equilibriums, let $\dot{x}_{1}=0$ and $\dot{x}_{2}=0$, leading to

$$
\bar{x}_{1}=\frac{1}{\alpha_{10}}\left(\frac{\beta_{10} \bar{x}_{2}}{1+\varepsilon_{0}\left|\bar{x}_{2}\right|}+F_{10}\right), \quad \bar{x}_{2}=\frac{1}{\alpha_{20}}\left(\frac{\beta_{20} \bar{x}_{1}}{1+\varepsilon_{0}\left|\bar{x}_{1}\right|}+F_{20}\right) .
$$

The two equations in (12) represents two curved nullclines, respectively. The fixed point $(\mathrm{s}) \overline{\boldsymbol{x}}_{i}^{\mathrm{T}}=\left[\bar{x}_{i 1}, \bar{x}_{i 2}\right]$ can easily be found as the intersection point(s) of the two lines. Since the equations determining the fixed points for each pair of $\bar{x}_{i 1}$ and $\bar{x}_{i 2}$ are quadratic, they can be calculated explicitly for both cases $\bar{x}_{i 1}, \bar{x}_{i 2}>0$ and $\bar{x}_{i 1}, \bar{x}_{i 2}<0$, respectively. It is found that a positive pair of $\bar{x}_{11}, \bar{x}_{12}>0$ exists for all conceivable parameter combinations. However, there may be two negative solution pairs, $\bar{x}_{21}, \bar{x}_{22}<0$ and $\bar{x}_{31}, \bar{x}_{32}<0$, depending on specific values of the system parameters. The latter two fix points may degenerate into one for some special combination of parameters.

In the linear case under condition (7), the two nullclines are degenerated into two straight lines, and only one intersection point exists, as shown in Fig. 1. This is an asymptotically stable fixed point in form of a stable node which is a global attractor. In the nonlinear case, however, condition (7) is not necessary, and a greater variety is possible depending on the values of parameters $\beta_{10}$ and $\beta_{20}$. The following are two typical cases.

The case of $\beta_{10} \beta_{20}<\alpha_{10} \alpha_{20}$ (i.e., Eq. (7) holds, is shown in Fig. 3a with the parameter set of Fig. 1 supplemented by $\varepsilon_{0}=0.2$ ). The two nullclines only intersect at one point in the first quadrant. Therefore, the number of attractors remains the same as in the linear case, i.e., there is only one stable node attractor in the first quadrant. The case is similar to the linear case qualitatively, and the couple is called a robust one.

For another choice of system parameters, the number of attractors may increase. Consider a case with parameters $\alpha_{10}, \alpha_{20}, F_{10}, F_{20}$, and $\varepsilon_{0}$ the same as in Fig. 3a, but $\beta_{10}=4, \beta_{20}=3$ so that Eq. (7) is violated, i.e. $\beta_{10} \beta_{20}>\alpha_{10} \alpha_{20}$. There exist three equilibriums, as shown in Fig. $3 \mathrm{~b}$. Two of them, $\overline{\boldsymbol{x}}_{1}$ and $\overline{\boldsymbol{x}}_{3}$, are stable nodes in the first and the third quadrants, characterizing one plateau of love, and another one of hate, respectively. The third attractor $\overline{\boldsymbol{x}}_{2}$ is an unstable saddle point between the two stable nodes, also in the third quadrant. Although the basin of attraction for the fixed point of love $\left(\overline{\boldsymbol{x}}_{1}\right)$ is larger than that for the plateau of hate $\left(\overline{\boldsymbol{x}}_{3}\right)$, there is some danger that the relationship ends in antagonism $\left(\overline{\boldsymbol{x}}_{3}\right)$. In this case, the relationship is a fragile one. 


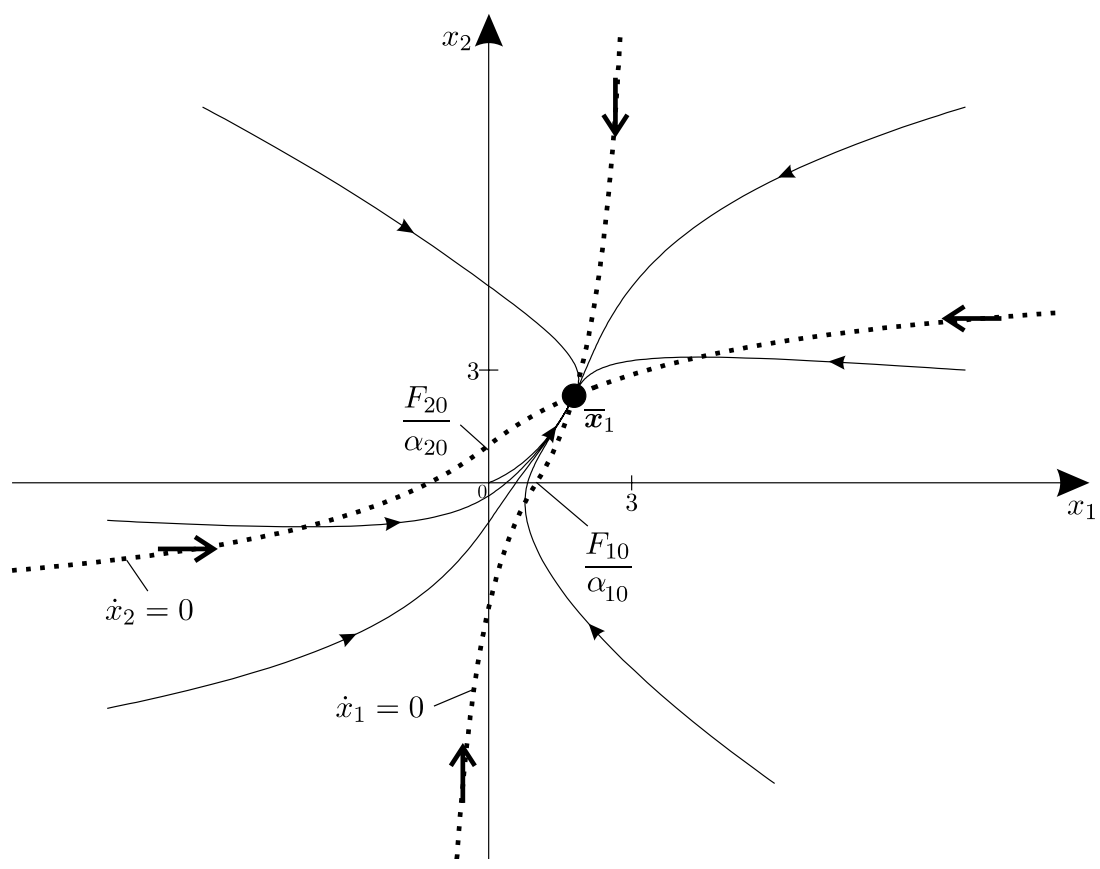

Fig. 3a. Evolution of the feelings in a robust couple for different initial conditions.

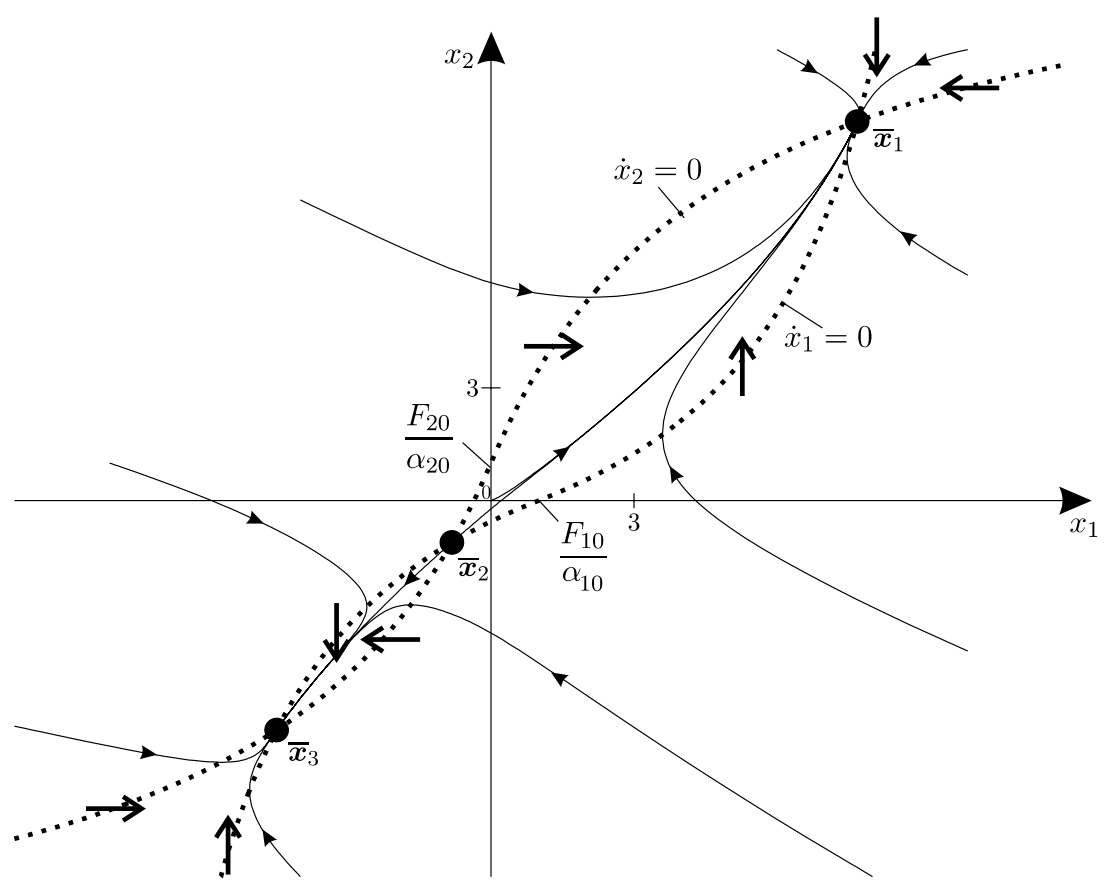

Fig. 3b. Evolution of the feelings in a fragile couple for different initial conditions.

It is noted that the represented portrait of a fragile couple, not discussed in [5,6], is a romantic style only for the case of nonlinear return functions and condition (7) does not hold. It is realistic, but could not be described by a linear model. 


\section{Linear model with time fluctuations}

We now return to the linear model Eqs. (4) and (5) but replace the input source terms $F_{i 0}$ by time-fluctuating $F_{i}(t)$ and replace the system parameters $\alpha_{i 0}, \beta_{i 0}$ by time-varying $\alpha_{i}(t)$ and $\beta_{i}(t)$, respectively. Both deterministic and stochastic variations of such terms will be considered. Furthermore, the two coupled first-order governing Eqs. (4) and (5) can be decoupled, leading to two independent second-order equations as follows:

$$
\ddot{x}_{i}+\left(\alpha_{1}+\alpha_{2}-\frac{\dot{\beta}_{i}}{\beta_{i}}\right) \dot{x}_{i}+\left(\alpha_{1} \alpha_{2}-\beta_{1} \beta_{2}+\dot{\alpha}_{i}-\alpha_{i} \frac{\dot{\beta}_{i}}{\beta_{i}}\right) x_{i}=\dot{F}_{i}+\left(\alpha_{j}-\frac{\dot{\beta}_{i}}{\beta_{i}}\right) F_{i}+\beta_{i} F_{j}, \quad j \neq i ; i, j=1,2 .
$$

Realistically, the appeal function $F_{i}$ and its derivative must be bounded since $F_{i}$ describes the reaction of the individual $i$ to the appeal of the partner $j$. We assume that the stability condition (7) is satisfied.

\subsection{Oscillating source terms}

First we consider that case in which the system parameters are constant, i.e., $\alpha_{i}(t)=\alpha_{i 0}, \beta_{i}(t)=\beta_{i 0}, i=1,2$, and only the source terms fluctuate. Eq. (13) are then simplified to

$$
\ddot{x}_{i}+\left(\alpha_{10}+\alpha_{20}\right) \dot{x}_{i}+\left(\alpha_{10} \alpha_{20}-\beta_{10} \beta_{20}\right) x_{i}=\dot{F}_{i}(t)+\alpha_{j 0} F_{i}(t)+\beta_{i 0} F_{j}(t), \quad j \neq i ; i, j=1,2 .
$$

Obviously, this are the equations of motion for two damped oscillators under external excitations with the same natural circular frequency

$$
\omega_{0}=\sqrt{\alpha_{10} \alpha_{20}-\beta_{10} \beta_{20}}
$$

of the corresponding undamped system and the same damping coefficient

$$
D=\frac{\alpha_{10}+\alpha_{20}}{2 \sqrt{\alpha_{10} \alpha_{20}-\beta_{10} \beta_{20}}} .
$$

It is clear that $D>1$ under the condition (7), and the oscillators in (14) are over-damped.

We assume harmonic fluctuations

$$
F_{i}(t)=F_{i 0}\left(1+\gamma_{i 0} \cos \Omega t\right), \quad i=1,2
$$

for the source terms where (for simplification) the rhythm, i.e., the circular frequency $\Omega$ is the same for both partners and a phase angle is not taken into consideration. It is straightforward to find the complete solutions

$$
\begin{aligned}
x_{i}(t)= & \mathrm{e}^{-D \omega_{0} t}\left(A_{i} \mathrm{e}^{\sqrt{D^{2}-1} \omega_{0} t}+B_{i} \mathrm{e}^{-\sqrt{D^{2}-1} \omega_{0} t}\right)+\frac{\alpha_{j 0} F_{i 0}+\beta_{i 0} F_{j 0}}{\alpha_{10} \alpha_{20}-\beta_{10} \beta_{20}}+\frac{\alpha_{j 0} F_{i 0} \gamma_{i 0}+\beta_{i 0} F_{j 0} \gamma_{j 0}}{\sqrt{\left(\omega_{0}^{2}-\Omega^{2}\right)^{2}+(2 D \Omega)^{2}}} \\
& \times \cos \left(\Omega t+\arctan \frac{2 D \Omega}{\omega_{0}^{2}-\Omega^{2}}\right)-\frac{F_{i 0} \gamma_{i 0} \Omega}{\sqrt{\left(\omega_{0}^{2}-\Omega^{2}\right)^{2}+(2 D \Omega)^{2}}} \sin \left(\Omega t+\arctan \frac{2 D \Omega}{\omega_{0}^{2}-\Omega^{2}}\right), \quad i=1,2
\end{aligned}
$$

for Eq. (14) where $A_{i}$ and $B_{i}$ are constants determined according to the initial conditions. Eq. (18) shows that the steady-state solutions are also harmonic functions with circular frequency $\Omega$. Fig. 4 depicts a trajectory for the system under the zero initial conditions with the same parameter values as in Fig. 1 , and $\gamma_{10}=\gamma_{20}=1$ and $\Omega / \omega_{0}=2$. After the transient terms are damped out, the steady-state trajectory is an elliptic orbit in the $x_{1}-x_{2}$ state plane. The center of the orbit is the fixed point $\bar{x}$ in Fig. 1. It should be noted that due to the strong damping (for the system data chosen, $D=1.5$ results), the magnitude of the stationary cycle which for $\Omega \rightarrow 0$ is the largest, decreases monotonically with increasing $\Omega$ without any pronounced resonance phenomenon and vanishes asymptotically when $\Omega \rightarrow \infty$. 


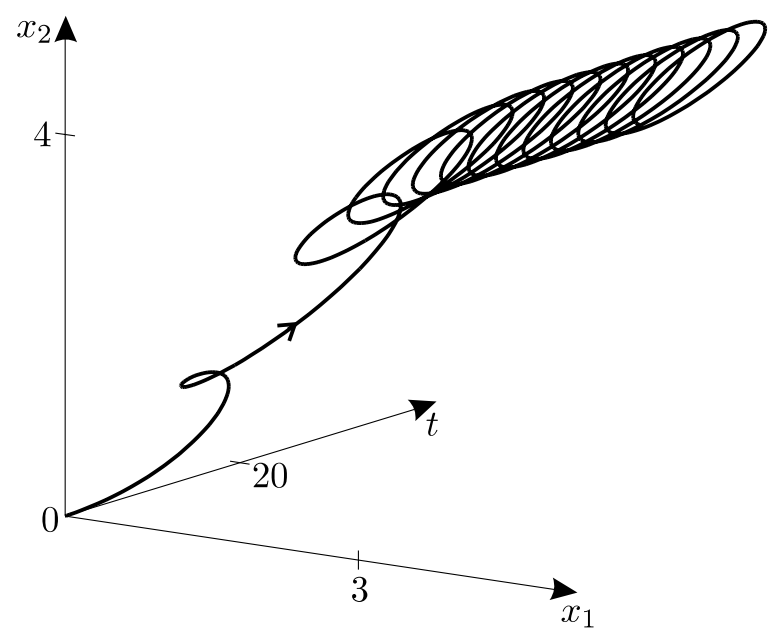

Fig. 4. Evolution of the feelings in a linear couple with harmonically fluctuating source terms.

\subsection{Oscillating system parameters}

If the appeal functions $F_{i}, i=1,2$ are constants, i.e., $F_{i}(t)=F_{i 0}$, but the system parameters are time-fluctuating, the governing second-order differential equations read

$$
\begin{aligned}
\ddot{x}_{i} & +\left(\alpha_{1}(t)+\alpha_{2}(t)-\frac{\dot{\beta}_{i}(t)}{\beta_{i}(t)}\right) \dot{x}_{i}+\left(\alpha_{1}(t) \alpha_{2}(t)-\beta_{1}(t) \beta_{2}(t)+\dot{\alpha}_{i}(t)-\alpha_{i}(t) \frac{\dot{\beta}_{i}(t)}{\beta_{i}(t)}\right) x_{i} \\
& =\left(\alpha_{j}(t)-\frac{\dot{\beta}_{i}(t)}{\beta_{i}(t)}\right) F_{i 0}+\beta_{i}(t) F_{j 0}, \quad j \neq i ; i, j=1,2 .
\end{aligned}
$$

Obviously, they are the equations of motion of damped oscillators under both parametric and external excitations. Assume the time variations of the system parameters $\alpha_{i}(t)$ and $\beta_{i}(t), i=1,2$ to be harmonic, i.e.,

$$
\begin{aligned}
& \alpha_{i}(t)=\alpha_{i 0}\left(1+\delta_{i 0} \cos \Omega t\right), \quad i=1,2, \\
& \beta_{i}(t)=\beta_{i 0}\left(1+\delta_{i 0} \cos \Omega t\right), \quad i=1,2 .
\end{aligned}
$$

For simplicity, the circular frequency here is assumed to be the same for all parameters. Even though, there are not longer mono-frequent fluctuations but periodic ones with an infinite number of harmonic components with frequencies in form of whole-numbered multiples of $\Omega$ as the basic one. Fig. 5 shows the trajectory of

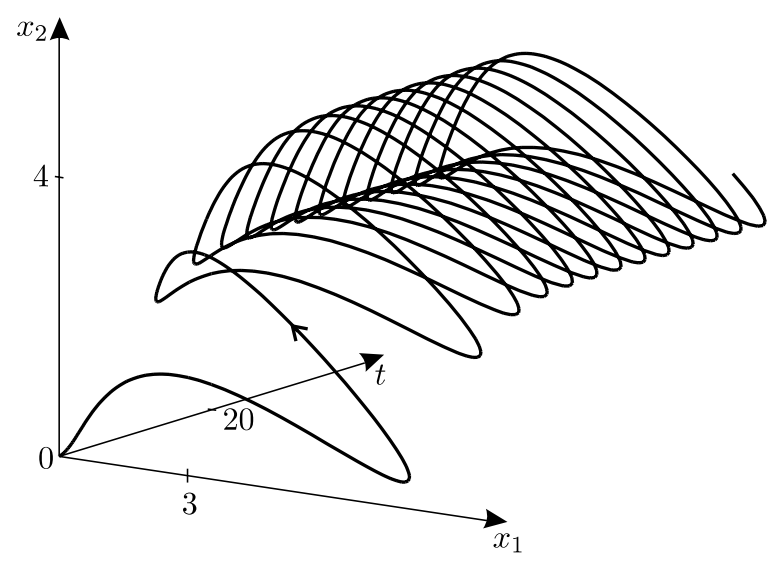

Fig. 5. Evolution of the feelings in a linear couple for harmonically fluctuating system parameters. 
the system evolution for the same parameters as in Fig. 1, with $\delta_{10}=\delta_{20}=2, \Omega / \omega_{0}=2$ and zero initial conditions. Obviously, the steady-state orbit differs from an elliptic one.

Taking into consideration that for the assumed cautious lovers, there is such a large damping involved that the free motions are creeping motions and not oscillations it becomes clear that for such a system parametricexcited vibrations as a typical instability of Hill's equation cannot appear. Introduced perturbations are decreasing and steady-state orbits in the $x_{1}, x_{2}$ phase space can only be induced by the appearing external excitation terms. It follows that for cautious lovers, variations in system parameters need not to be considered if only the qualitative dynamic behavior is to be investigated.

\subsection{Oscillating source terms and system parameters}

In the previous two sections, the harmonic fluctuations are assumed in either the source terms or the system parameters. For the more complicated case where the variations appear in both the source terms and the system parameters, the result is shown in Fig. 6 with the same parameter values as in Figs. 4 and 5. It is seen that the steady-state trajectory is similar to that of Fig. 5.

For simplicity, only one circular frequency $\Omega$ is assumed for all harmonic fluctuations. In reality, different partners have different periods in love feelings, and the periods are also different for the oblivion $\alpha_{i}(t)$, the return $\beta_{i}(t)$ and the appeal $F_{i}(t)$. It is also noted that no combination of different harmonic functions is considered in the above cases, and the treatment is analogous if the fluctuations are periodic instead harmonic.

\subsection{Random fluctuations in both source terms and system parameters}

To take random disturbance into consideration, the following stochastic process is introduced

$$
\xi(t)=\cos \theta(t), \quad \mathrm{d} \theta=\Omega \mathrm{d} t+\sigma \mathrm{d} B(t),
$$

where $\Omega$ and $\sigma$ are positive constants, and $B(t)$ is a unit Wiener process. By adding a random phase in the harmonic process, the stochastic process $\xi(t)$ is called the randomized harmonic process. This randomized harmonic process was proposed independently by Dimentberg [1] and Wedig [11], and can be used to model a variety of random phenomena. It reduces to a pure sinusoidal signal when $\sigma=0$. Parameter $\Omega$ gives the peak location of the spectral density, and $\sigma$ represents the level of stochasticity. The process has a narrow peak when $\sigma$ is small. As $\sigma$ increases, the spectral density becomes increasingly flat.

Now the stochastic process $\xi(t)$ can be used to replace the harmonic process $\cos \Omega t$ in the corresponding state equations for a variety of situations to investigate effects of the random fluctuations. For randomly fluctuating source terms as well as the system parameters, for instance, we have the equations of motion

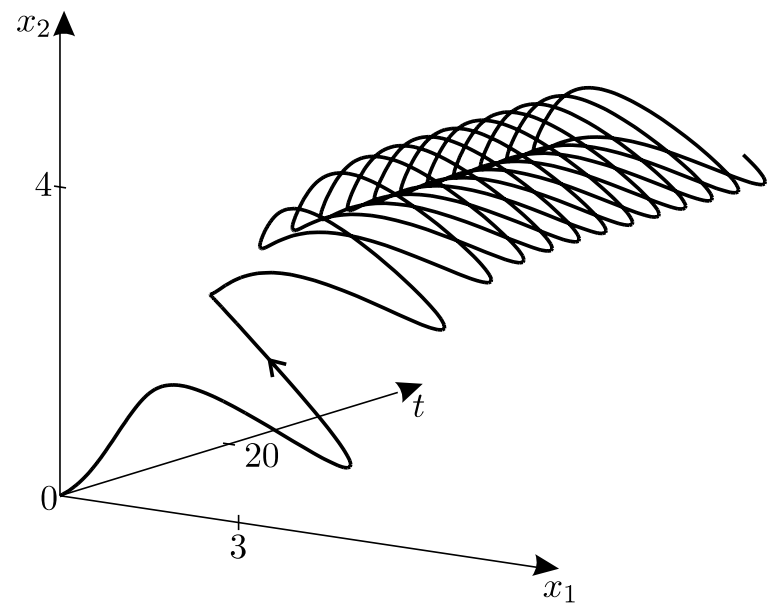

Fig. 6. Evolution of the feelings in a linear couple with both harmonically fluctuating source terms and system parameters. 


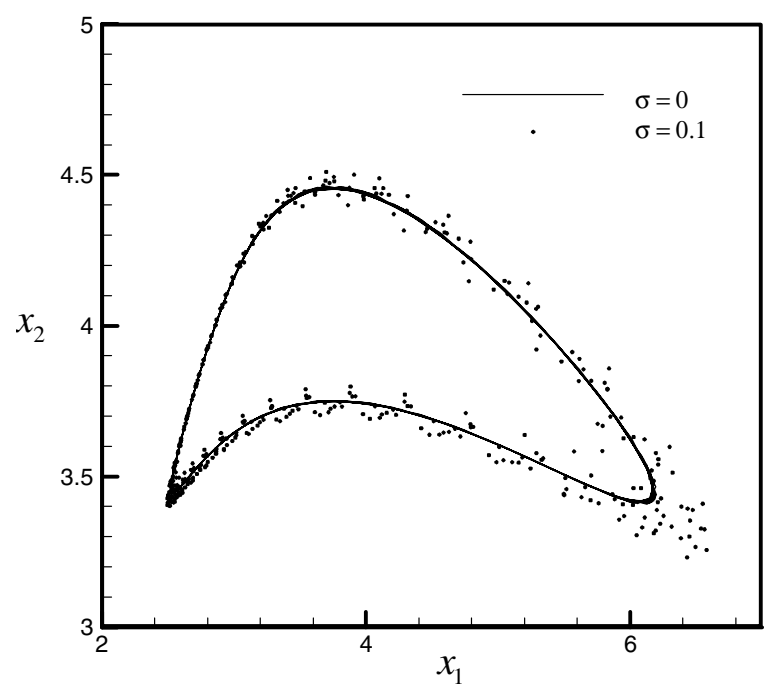

Fig. 7. Steady-state feelings of a linear couple with weak random fluctuations.

$$
\begin{aligned}
& \dot{x}_{1}=-\alpha_{10}\left[1+\delta_{10} \xi(t)\right] x_{1}+\beta_{10}\left[1+\delta_{10} \xi(t)\right] x_{2}+F_{10}\left[1+\gamma_{10} \xi(t)\right], \\
& \dot{x}_{2}=-\alpha_{20}\left[1+\delta_{20} \xi(t)\right] x_{2}+\beta_{20}\left[1+\delta_{20} \xi(t)\right] x_{1}+F_{20}\left[1+\gamma_{20} \xi(t)\right],
\end{aligned}
$$

where $\xi(t)$ is given by relation (22). Monte Carlo simulations were carried out to investigate system behaviors for the stochastic system, Eqs. (23) and (24), while keeping the system parameters the same as those when obtaining Figs. 4 and 5, namely, $\alpha_{10}=2, \alpha_{20}=1, \beta_{10}=\beta_{20}=1, \delta_{10}=\delta_{20}=2, \gamma_{10}=\gamma_{20}=1, F_{10}=2$, $F_{20}=1$, and $\Omega=2 \omega_{0}$. Fig. 7 shows results obtained for the two cases of $\sigma=0$ and $\sigma=0.1$, respectively. The case of $\sigma=0$ corresponds to a harmonic disturbance, and the result shown in the figure is the steady-state trajectory and is periodic. In the case of $\sigma=0.1$, corresponding to weak stochasticity, the love relationship deviates from that of the periodic trajectory, and forms a distribution of relationship. At a higher stochasticity level of $\sigma=0.5$, the deviation is much wider, as shown in Fig. 8. At some time instants, the change of feeling of each partner may be quite dramatic, and the change is not exactly predictable. In general, cautious lovers have lower stochasticity level.

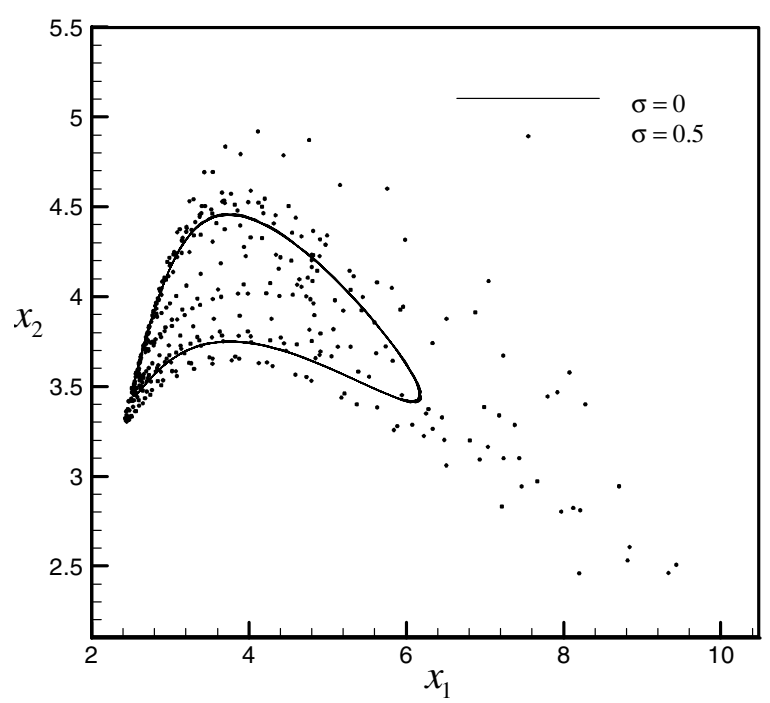

Fig. 8. Steady-state feelings in a linear couple with strong random fluctuations. 


\section{Nonlinear model with time fluctuations}

In general, no analytical solution can be found when the return functions are nonlinear. Assuming small nonlinearities $\varepsilon_{0} \ll 1$ in Eq. (9), the nonlinear return function $R_{i}$ can then be approximated as

$$
R_{i}\left(x_{j}\right)=x_{j}\left(1-\varepsilon_{0}\left|x_{j}\right|\right), \quad i, j=1,2 .
$$

In this case, a perturbation analysis can be performed to compute solutions originating from those of the linear case. The resulting co-domain of the variables $x_{j}, j=1,2$, is not allowed to exceed a value of about $x_{j} \approx 1 /\left(2 \varepsilon_{0}\right)$ because only for $x_{j}(t) \leqslant 1 /\left(2 \varepsilon_{0}\right)$ an increasing absolute value of the return function is ensured. It follows that for an accurate perturbation analysis, the absolute values of the fixed point coordinates have to be restricted to numbers not significantly greater than these limiting values. On the other hand, the system is strongly damped for cautious robust lovers, so that - this is well-known from the discussion of corresponding Duffing oscillators - the difference between the linear and the nonlinear models may be insignificant using perturbation analysis.

In cases where the absolute values of the fixed point coordinates exceed the given limiting values, a perturbation analysis is no longer applicable, and only numerical simulations are promising. Such simulations are straightforward for deterministically fluctuating source terms and system parameters, due to the low system order. Results are shown in Fig. 9 for the nonlinear model under harmonically oscillating source terms with parameters of Fig. 3a, and with $\gamma_{10}=\gamma_{20}=1$ and $\Omega / \omega_{0}=2$. As shown in Fig. 9, the solution for the harmonic input is periodic, but no longer harmonic due to the nonlinearity (for the data chosen the effect is small). The center of the steady-state orbit in Fig. 9 is expected to coincide approximately with the fixed point $\overline{\boldsymbol{x}}_{1}$ in Fig. 3a. Except that the solution is no longer harmonic, no further significant effects of the nonlinear return function are observable.

For the case of a fragile couple, for which condition (7) does not hold, the steady-state orbit under harmonically oscillating source terms may be about either equilibrium point $\overline{\boldsymbol{x}}_{1}$ or $\overline{\boldsymbol{x}}_{3}$, depending on the initial conditions. Fig. 10 shows two different trajectories corresponding to two different initial conditions for the same system parameters as those in Fig. $3 b$, and with $\gamma_{10}=\gamma_{20}=1$ and $\Omega / \omega_{0}=2$. The initial conditions $x_{1}(0)=0$ and $x_{2}(0)=-1.656$ lead to a steady-state orbit about $\overline{\boldsymbol{x}}_{1}$; thus the couple ends in love. The initial conditions $x_{1}(0)=-0.95$ and $x_{2}(0)=-0.95$ lead to another steady-state orbit near $\overline{\boldsymbol{x}}_{3}$, indicating that finally, the couple is hating each other.

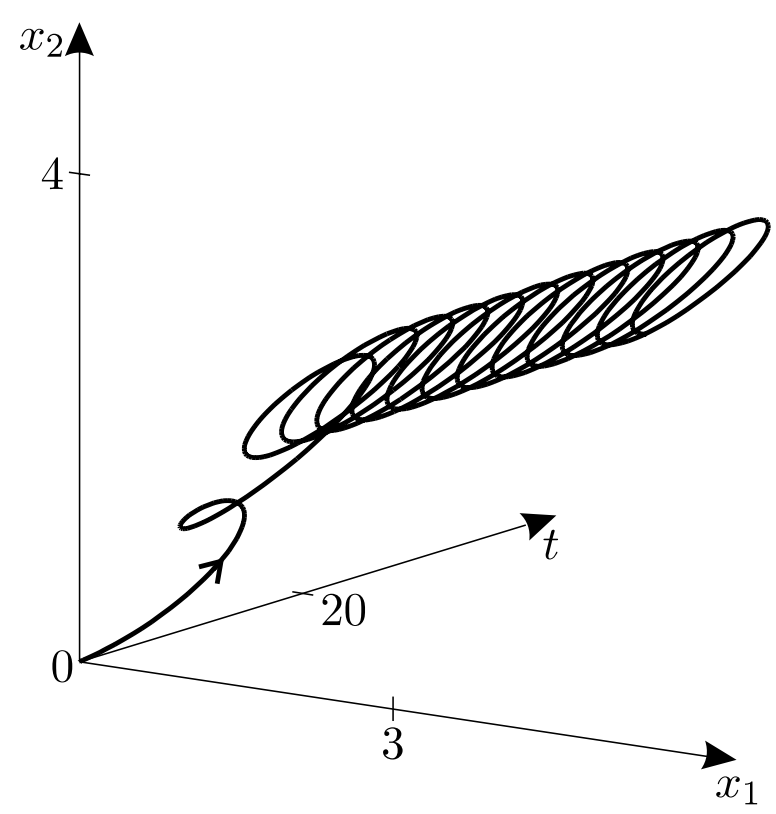

Fig. 9. Evolution of the feelings in a nonlinear robust couple with harmonically fluctuating source terms. 


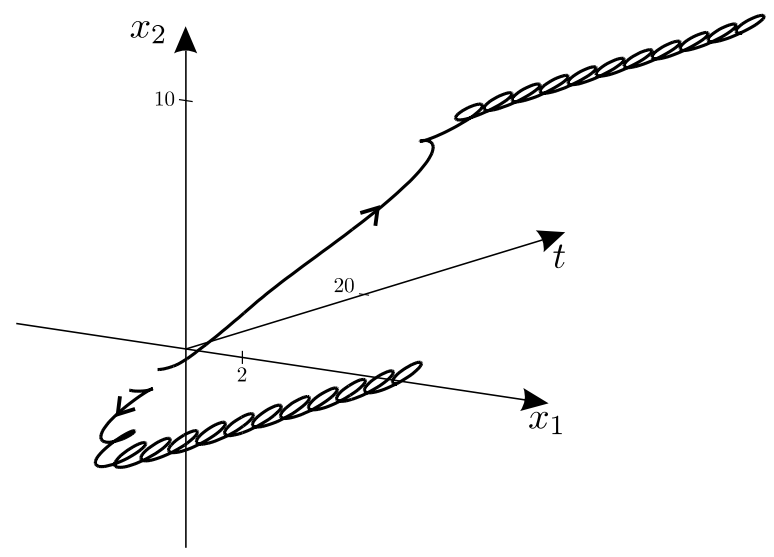

Fig. 10. Evolution of the feelings in a nonlinear fragile couple with harmonically fluctuating source terms.

Similar phenomena can be found for more complex deterministic fluctuations in the system parameters, or in both the source terms and system parameters.

For the case of nonlinear return functions and stochastic disturbances in both the source terms and the system parameters, the linear return functions $\beta_{10} x_{2}$ in Eq. (23) and $\beta_{20} x_{1}$ in Eq. (24) are replaced by the nonlinear ones given in Eq. (9). Figs. 11 and 12 show stationary trajectories for a nonlinear robust couple with parameters of Fig. 3a, and $\gamma_{10}=\gamma_{20}=1, \delta_{10}=\delta_{20}=2$ and $\Omega / \omega_{0}=2$. The centers of the steady-state orbits in Figs. 11 and 12 coincide approximately with the fixed point $\overline{\boldsymbol{x}}_{1}$ in Fig. 3a. Similar effects of the stochastic disturbance are observed as those in the linear case. A higher stochasticity of $\sigma=0.5$ causes the love relation ship more unstable, as shown in Fig. 12. However, the love relationship is still in place. Similar to the case of harmonic fluctuations, no significant effects of the nonlinear return functions are found.

The situation is more complicated for a fragile couple under random disturbance. Beginning from the same initial conditions, the outcomes may be very much different if random disturbances are present. Fig. 13 shows two different trajectories for the same system with parameters same as those in Fig. $3 \mathrm{~b}$, with $\gamma_{10}=\gamma_{20}=1$, $\delta_{10}=\delta_{20}=2$, and under the same initial conditions of $x_{1}(0)=0$ and $x_{2}(0)=-1.656$ as in Fig. 10. The two steady-state trajectories are totally different. One is around the attraction point $\overline{\boldsymbol{x}}_{1}$, and the couple is in love relationship. The other is around the attraction point $\overline{\boldsymbol{x}}_{3}$, and the couple ends in hate relationship. Therefore, a random event may cause a relationship to deteriorate.

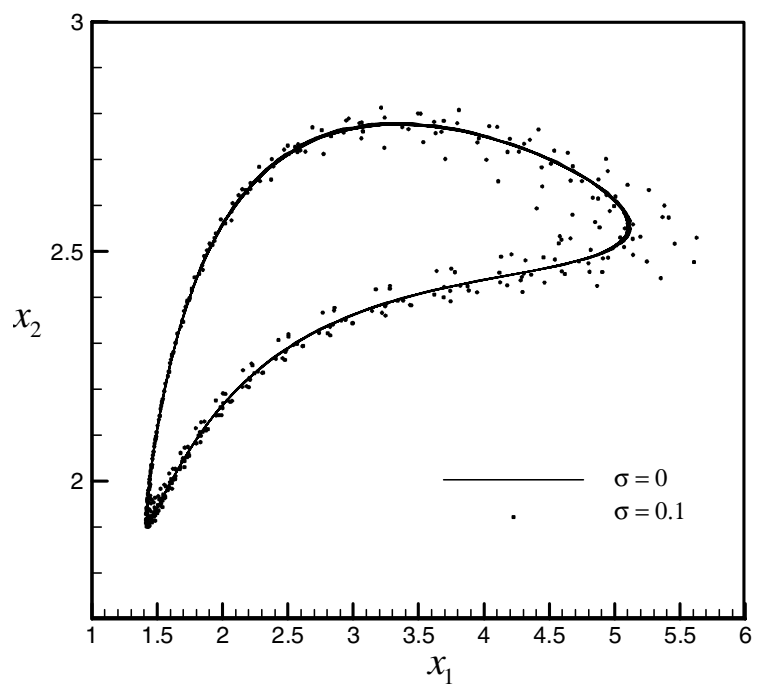

Fig. 11. Steady-state feelings of a nonlinear robust couple with weak random fluctuations. 


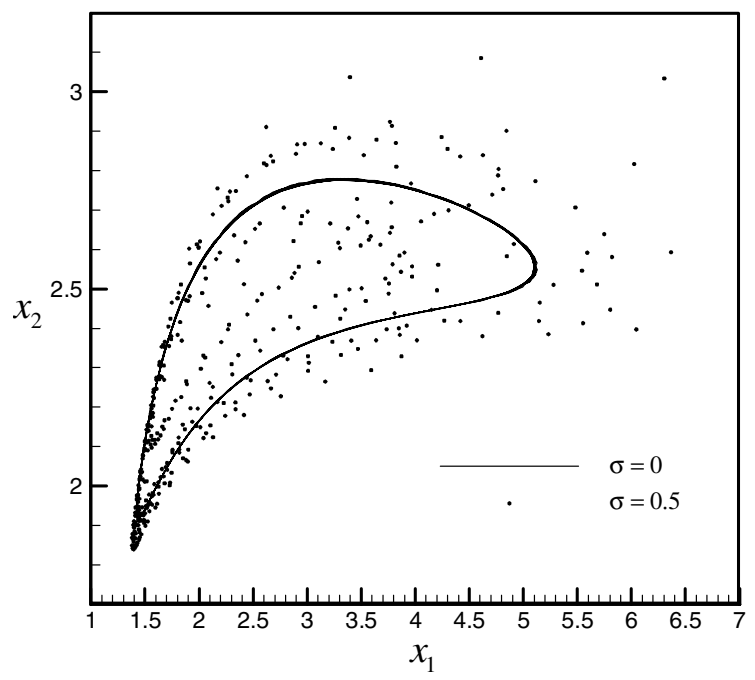

Fig. 12. Steady-state feelings in a nonlinear robust couple with strong random fluctuations.

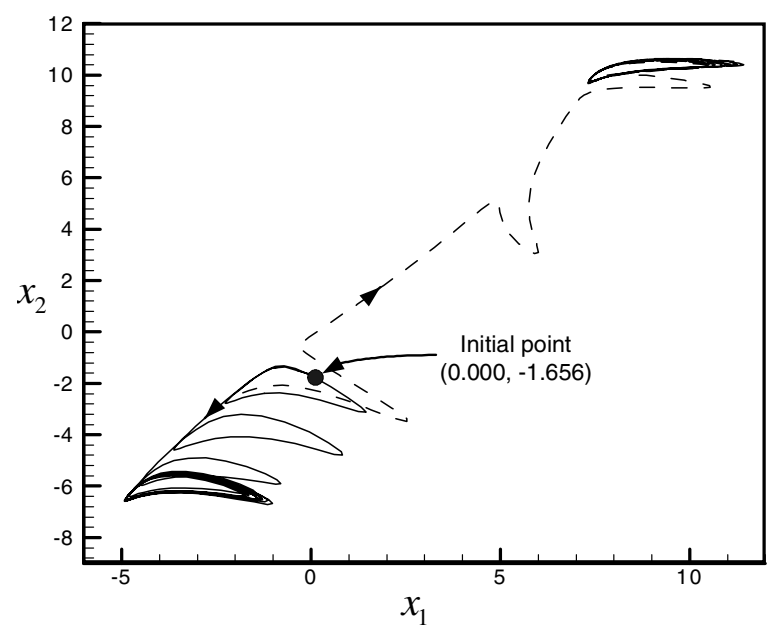

Fig. 13. Two possible outcomes of a nonlinear fragile couple with random fluctuations.

\section{Discussion}

The dynamics of romantic relationships of two individuals are significantly more complex when time fluctuations of source terms and system parameters are taken into consideration. It is clear that time-independent personalities and appeals are an academic idealization. In reality, there are short- and long-termed fluctuations of personal feelings due to, for instance, biological cycles and varying stresses from the daily job. While it is straightforward to introduce such an emotional patterns into the mathematical model, it is difficult to measure parameter values quantitatively. For couples with all kinds of exotic feeling, a never-ending list of new phenomena may be expected. However, the variability is to be expected to be more limited for couples of cautious individuals. One interesting effect is the cyclic change of love about a stationary center of steady love for a couple governed by linear state equations, or a so-called robust couple governed by equations with nonlinear return functions. For a fragile couple, there is a second stable fixed point of stationary hate, and the cyclic behavior may occur about either of the two centers. A more complex irregular motion pattern surrounding 
both centers with alternating love and hate of different time spans might appear during different time periods, but it seems very seldom for cautious lovers with strong damping.

Closing the discussion, it may be noted that the examination of love triangles with time-varying fluctuations is another example of particular interest. Assume that individual 1 has feeling for two other individuals 2 and 3 , who do not know each other. Then the simplest mathematical model for such a case would be four-dimensional (see [7]),

$$
\begin{aligned}
& \dot{x}_{12}=-\alpha_{1}(t) x_{12}+\beta_{1}(t)\left(x_{2}-x_{3}\right)+F_{1}(t), \\
& \dot{x}_{2}=-\alpha_{2}(t) x_{2}+\beta_{2}(t) x_{12}+F_{2}(t), \\
& \dot{x}_{13}=-\alpha_{1}(t) x_{13}+\beta_{1}(t)\left(x_{3}-x_{2}\right)+F_{1}(t), \\
& \dot{x}_{3}=-\alpha_{3}(t) x_{3}+\beta_{3}(t) x_{13}+F_{3}(t) .
\end{aligned}
$$

It is obvious that the feeling of $x_{1}$ for $x_{2}\left(x_{3}\right)$ is weakened by the feeling of $x_{1}$ for $x_{3}\left(x_{2}\right)$, More complicated linear and nonlinear models are possible, and rich phenomena can be discovered.

\section{Concluding remarks}

Romantic relationships of cautious lovers are discussed when time varying effects are included. The feelings are modeled in form of two state equations with time varying source terms or/and system parameters. Both harmonic and random time fluctuations are considered. While analytical results can be obtained for harmonically fluctuating source terms (and system parameters) within the linear formulation, numerical simulation must be carried out for the other situations. The results show that linear and nonlinear robust couples of cautious individuals with time-fluctuating feelings tend to a cyclic emotional behavior about a fixed point of love while for nonlinear fragile couples, more complex patterns of emotional feelings are possible.

\section{Acknowledgement}

The first author is grateful to Professor Lin who invited him for a sabbatical stay in the Center of Applied Stochastics Research at the Florida Atlantic University where most of the work was performed.

\section{References}

[1] M.F. Dimentberg, Statistical Dynamics of Nonlinear Time-Varying Systems, Wiley, NY, 1988.

[2] J.M. Gottman, J.D. Murray, C.C. Swanson, R. Tyson, K.R. Swanson, The Mathematics of Marriage, MIT Press, MA, 2002.

[3] A. Gragnani, S. Rinaldi, G. Feichtinger, Cyclic dynamics in romantic relationships, Int. J. Bifurcat. Chaos 7 (1997) $2611-2619$.

[4] A. Rapoport, Fights, Games and Debates, Michigan Press, MI, 1960.

[5] S. Rinaldi, Love dynamics: the case of linear couples, Appl. Math. Comp. 95 (1998) 181-192.

[6] S. Rinaldi, A. Gragnani, Love dynamics between secure individuals: a modeling approach, Nonlinear Dyn. Psych. Life Sci. 2 (1998) 283-301.

[7] J.C. Sprott, Dynamical models of love, Nonlinear Dyn. Psych. Life Sci. 8 (2004) 303-314.

[8] J.C. Sprott, Dynamical models of happiness, Nonlinear Dyn. Psych. Life Sci. 9 (2005) 23-36.

[9] S.H. Strogatz, Love affairs and differential equations, Math. Mag. 61 (1988) 35.

[10] S.H. Strogatz, Nonlinear Dynamics and Chaos. With Applications to Physics, Biology, Chemistry, and Engineering, Springer, NA, 1994.

[11] W.V. Wedig, Analysis and simulation of nonlinear stochastic system, in: W. Schiehlen (Ed.), Nonlinear Dynamics in Engineering Systems, Springer, Germany, 1989. 